\title{
Effective motion of a curvature-sensitive interface through a heterogeneous medium
}

\author{
BOGDAN CRACIUN ${ }^{\dagger}$ \\ Synopsys, Inc., 700 E. Middlefield Road, Mountain View, CA 94043-4033, USA \\ AND \\ KAUSHIK BHATTACHARYA \\ Mail Stop 104-44, California Institute of Technology, Pasadena, CA 91125, USA
}

[Received 19 August 2001 and in revised form 28 January 2004]

\begin{abstract}
This paper deals with the evolution of fronts or interfaces propagating with normal velocity $v_{n}=$ $f-c \kappa$, where $f$ is a spatially periodic function, $c$ a constant and $\kappa$ the mean curvature. This study is motivated by the propagation of phase boundaries and dislocation loops through heterogeneous media. We establish a homogenization result when the scale of oscillation of $f$ is small compared to the macroscopic dimensions, and show that the overall front is governed by a geometric law $v_{n}=\bar{f}(n)$. We illustrate the results using examples. We also provide an explicit characterization of $\bar{f}$ in the limit $c \rightarrow \infty$.
\end{abstract}

\section{Introduction}

This paper deals with the evolution of fronts or interfaces propagating with a normal velocity that depends on the position and the mean curvature of the front. Specifically consider a front propagating with normal velocity

$$
v_{n}=f-c \kappa,
$$

where $f=f(x)$ is a given spatially dependent function, $c$ is a constant and $\kappa$ is the mean curvature. Suppose $f$ is heterogeneous on a scale small compared to the domain; then the evolution of the front may be very complicated. It is of interest then to ask if one can define an average or overall frontone that captures the essential macroscopic features and ignores the exact microscopic details—and describe its propagation. That is the issue studied in this paper.

A motivation for such a study is the motion of a phase boundary through a heterogeneous material, for example a matrix with precipitates. Consider a body occupying a region $\Omega \subset \mathbb{R}^{N}$ consisting of one phase occupying a region $\mathcal{A} \subset \Omega$ and surrounded by a second phase which occupies $\Omega \backslash \mathcal{A}$. Phase transformation causes the region $\mathcal{A}$ to evolve, and we are interested in this evolution. The energy associated with the two-phase arrangement is

$$
\mathcal{E}(\mathcal{A})=\int_{\mathcal{A}} \phi^{+}(x) \mathrm{d} x+\int_{\Omega \backslash \mathcal{A}} \phi^{-}(x) \mathrm{d} x+\int_{\partial \mathcal{A}} c \mathrm{~d} a,
$$

\footnotetext{
†Email: bogdan@synopsys.com

Corresponding author. Email: bhatta@ caltech.edu
} 
where $\phi^{ \pm}$is the density of the bulk energy in the two phases and $c$ is the density of the interfacial energy $(c>0)$. We assume that the bulk energy density may depend on position in view of inhomogeneities and defects in the material. We assume that the interfacial energy density is constant for simplicity. The energy dissipation associated with the motion of the phase boundary can be shown (following Gurtin [21]) to be

$$
\triangle(\mathcal{A})=\int_{\partial \mathcal{A}}(f-c \kappa) v_{n} \mathrm{~d} a,
$$

where $-f=\phi^{+}-\phi^{-}$is the jump in the bulk energy density across the phase boundary, $v_{n}$ is the normal velocity of the interface and $\kappa$ is the mean curvature. Therefore, $f-c \kappa$ is the thermodynamic force conjugate to the normal velocity and may be interpreted as the thermodynamic driving force that drives the phase boundary. It is natural, therefore, to assume that the normal velocity depends on the driving force, and (1.1) is the simplest model of this type. We note that this model automatically satisfies the requirement that the dissipation be non-negative.

A specialization of this interfacial evolution model is the Edwards-Wilkinson model much studied in statistical mechanics (see for example [22]). We may obtain this model from our equation (1.1) by assuming that the interface is a graph, linearizing the curvature and taking $f$ to be stochastic (constant plus an appropriate noise). The equation 11.1 is also often used in $\mathbb{R}^{2}$ to study the evolution of dislocation loops, where it is referred to as the 'line tension model' (see for example [24]).

There is large literature on (1.1) when $f$ is constant that establishes existence [9] and proposes fast numerical algorithms (see for example [31, 30]). However, actual materials often contain defects and heterogeneities, and therefore $f$ is not constant. These defects and heterogeneities can change the evolution significantly, and thus have a critically important implication in practice. Therefore the role of defects have also been studied in a variety of settings. Notable among them is the study of dislocation loops in material science where $f$ is assumed to be either constant except on a collection of small inclusions (where it takes another value) or a collection of point defects (Dirac masses). The motion past an individual defect and the "bow-out" of dislocation loops pinned by multiple defects is well understood [24]. More recently, numerical studies that consider a very large number of (periodically or randomly distributed) defects and also take into account the elastic interactions have been conducted [17, 25, 23, 27, 14]. Another interesting literature uses statistical mechanics to study the Edwards-Wilkinson and related models of interfaces propagating in a random medium (see for example [22, 28, 2]). A related literature concerns the propagation of contact line in the presence of defects [20, 32].

Yet, many important and interesting questions remain open. In particular, a mathematical treatment from the homogenization viewpoint is missing. We develop it in this paper, and obtain interesting and useful insights. This paper is the second in a series dealing with the propagation of fronts through heterogeneous media. The first [11] considered only $f$ and ignored the curvature. The third [12] considers the situation where the evolution of the interface is coupled to a partial differential equation in $\Omega$ (specifically elasticity).

An efficient tool for studying problems such as 1.1 is the level set formulation. If we assume that there exists a smooth function $h: \mathbb{R}^{N} \times[0, T) \rightarrow \mathbb{R}$ such that our front coincides with its zero level set at all times, a simple calculation yields

$$
n=\frac{\nabla h}{|\nabla h|}, \quad \kappa=\operatorname{div} \frac{\nabla h}{|\nabla h|}, \quad v_{n}=-\frac{1}{|\nabla h|} \frac{\partial h}{\partial t} ;
$$


so our normal velocity law (1.1) implies that $h$ satisfies the following equation:

$$
\frac{\partial h}{\partial t}=-f(x)|\nabla h|+c|\nabla h| \operatorname{div} \frac{\nabla h}{|\nabla h|} .
$$

To this equation one has to attach the initial condition

$$
h(x, 0)=h_{0}(x) \quad \text { in } \mathbb{R}^{N},
$$

where $h_{0}$ is a function chosen such that its zero level set coincides with the initial position of the front, and appropriate boundary or far field conditions.

If the medium in which the front is propagating is periodic with unit cell $[0, \varepsilon]^{N}$, the corresponding problem is

$$
\begin{cases}\frac{\partial h^{\varepsilon}}{\partial t}=-f\left(\frac{x}{\varepsilon}\right)\left|\nabla h^{\varepsilon}\right|+\varepsilon c\left|\nabla h^{\varepsilon}\right| \operatorname{div} \frac{\nabla h^{\varepsilon}}{\left|\nabla h^{\varepsilon}\right|} & \text { in } \mathbb{R}^{N} \times[0, T), \\ h^{\varepsilon}(x, 0)=h_{0}(x) & \text { in } \mathbb{R}^{N},\end{cases}
$$

with $f$ continuous and periodic with period $Y_{N}=[0,1]^{N}$. Our aim is to study the homogenization of this phenomenon, i.e., to capture its limit behavior when the structure of the medium becomes infinitely fine $(\varepsilon \rightarrow 0)$.

We remark that the scaling of the curvature coefficient, $\varepsilon c$, chosen in $(1.3)$ is the most interesting. Heuristically, in this situation we expect the curvature to scale as $1 / \varepsilon$, or the radius of curvature of the interface to be comparable to the unit cell. So the curvature and heterogeneities contribute comparably to the evolution of the interface and thus interact. If we assume that the curvature coefficient is larger, or $\varepsilon^{\alpha} c$ for $\alpha<1$, the radius of curvature scales as $1 / \varepsilon^{\alpha}$ and becomes large compared to the unit cell as $\varepsilon \rightarrow 0$. In other words, the curvature term dominates so that the interface becomes flat and we obtain a one-dimensional problem studied in [5]. Similarly, if we assume that the curvature coefficient is smaller, or $\varepsilon^{\alpha} c$ for $\alpha>1$, then the interface does not feel the contribution of the curvature and one obtains geometric motion studied in [11].

We show in Section 2, following the methods of Evans [16], that, as $\varepsilon \rightarrow 0$, the solution of (1.3) converges uniformly to the solution of

$$
\begin{cases}\frac{\partial h}{\partial t}=-\bar{f}\left(\frac{\nabla h}{|\nabla h|}\right)|\nabla h| & \text { in } \mathbb{R}^{N} \times[0, T), \\ h(x, 0)=h_{0}(x) & \text { in } \mathbb{R}^{N},\end{cases}
$$

where $\bar{f}$ is determined by solving a suitable periodic problem on the unit cell 2.19 . This implies that the average or overall interface propagates with normal velocity

$$
v_{n}=\bar{f}(n),
$$

where $n$ is the normal to the interface. So, the normal velocity depends on the orientation of the interface but not on the position or the mean curvature. In short, the average interface is governed by an anisotropic geometric law.

We also show that if $f$ is strictly positive (negative), then the resulting $\bar{f}$ is also strictly positive (negative). If $f$ takes both signs, then $\bar{f}$ may be positive, negative or even zero. This corresponds to the interface being trapped. We explore this and other issues with various examples in Section 3.

We notice from our examples that the effective behavior is easily characterized when the curvature coefficient $c$ is large. We study this limit in Section 4 and provide an explicit characterization for $\bar{f}$ in this case. 


\section{A homogenization result}

This section considers the homogenization of (1.3) assuming that $c>0$. The case $c=0$ when 1.3 reduces to a Hamilton-Jacobi equation has been treated in [11, 15, 16, 26]. It has been shown that the viscosity solution of the Hamilton-Jacobi initial value problem

$$
\begin{cases}\frac{\partial h^{\varepsilon}}{\partial t}+f\left(\frac{x}{\varepsilon}\right)\left|\nabla h^{\varepsilon}\right|=0 & \text { in } \mathbb{R}^{N} \times[0, \infty), \\ h^{\varepsilon}(x, 0)=h_{0}(x) & \text { in } \mathbb{R}^{N},\end{cases}
$$

converges uniformly on $\mathbb{R}^{N} \times[0, T$ ) (for all $T<\infty$ ) to the viscosity solution of the homogenized Hamilton-Jacobi initial value problem

$$
\begin{cases}\frac{\partial h}{\partial t}+\bar{f}(\nabla h)|\nabla h|=0 & \text { in } \mathbb{R}^{N} \times[0, \infty), \\ h(x, 0)=h_{0}(x) & \text { in } \mathbb{R}^{N},\end{cases}
$$

for $\bar{f}$ determined by the solution of a suitable 'unit cell' problem. Various variational characterizations for $\bar{f}$ are given in [11].

We will in fact consider the homogenization of a slightly more general problem than 1.3 . Note that (1.3) can also be written as

$$
\frac{\partial h^{\varepsilon}}{\partial t}+F\left(\varepsilon \nabla^{2} h^{\varepsilon}, \nabla h^{\varepsilon}, \frac{x}{\varepsilon}\right)=0
$$

where

$$
F(A, p, x)=f(x)|p|-c\left\langle I-\frac{p \otimes p}{|p|^{2}}, A\right\rangle .
$$

The operator $F$ defined above is degenerate elliptic, in the sense that

$$
F(X, p, x) \geqslant F(Y, p, x) \text { if } Y \geqslant X \text { (i.e. if } Y-X \text { is positive semidefinite). }
$$

Also, it can be easily checked that $F$ is a geometric operator, in the sense that it has the following scaling invariance:

$$
F(\lambda X+\sigma p \otimes p, \lambda p, x)=\lambda F(X, p, x) \quad \text { for all } \lambda>0, \sigma \in \mathbb{R} .
$$

We now study the homogenization of the problem

$$
\begin{cases}\frac{\partial u^{\varepsilon}}{\partial t}+F\left(\varepsilon \nabla^{2} u^{\varepsilon}, \nabla u^{\varepsilon}, \frac{x}{\varepsilon}\right)=0 & \text { in } \mathbb{R}^{N} \times[0, T), \\ u^{\varepsilon}(x, 0)=u_{0}(x) & \text { in } \mathbb{R}^{N},\end{cases}
$$

where $F$ is a geometric and degenerate elliptic operator, Lipschitz continuous and periodic in the variable $x$ with unit cell $Y_{N}=[0,1]^{N}$. This more general problem includes our front propagation problem 1.3 as a particular case.

We start by gaining some insight with a formal asymptotic treatment. For this, it is natural to begin with the expansion (see [4] for a systematic presentation of such ansatz)

$$
u^{\varepsilon}(x, t)=u^{0}(x, t)+\varepsilon u^{1}\left(\frac{x}{\varepsilon}, t\right)+o(\varepsilon) .
$$


Plugging this expansion into 2.2 and identifying the terms in front of powers of $\varepsilon^{0}$, we find

$$
u_{t}^{0}(x, t)+F\left(\nabla_{y}^{2} u^{1}(y, t), \nabla_{x} u^{0}(x, t)+\nabla_{y} u^{1}(y, t), y\right)=0,
$$

where $y=x / \varepsilon$. This can be looked at as a partial differential equation for the corrector $u^{1}$; its solvability condition provides a constraint between the partial derivatives of the average $u^{0}$ :

$$
u_{t}^{0}+\bar{F}\left(\nabla u^{0}\right)=0,
$$

with $\bar{F}$ determined by the condition that a periodic solution $v$ of

$$
F\left(\nabla_{y}^{2} v(y), p+\nabla_{y} v(y), y\right)=\bar{F}(p)
$$

exists.

We now prove the homogenization theorem for [2.2], following the work of Evans [16].

THEOREM 1 Assume that $F$ is a degenerate elliptic and geometric operator, Lipschitz continuous on $S_{N} \times\left(\mathbb{R}^{N} \backslash\{0\}\right) \times[0, T)$, periodic in the variable $x$ with unit cell $Y_{N}=[0,1]^{N}$ and satisfying

$$
\lim _{p \rightarrow 0, X \rightarrow 0} \sup _{x}|F(X, p, x)|=0 .
$$

Further, assume that the viscosity solution of the problem

$$
\delta u+F\left(\nabla^{2} u, \nabla u+p_{0}, x\right)=0
$$

is Hölder continuous in $x$ and its Hölder coefficient is bounded with respect to $\delta$ for $\delta \in(0,1)$ (see Remark 2 after the theorem for a discussion on this assumption).

Then the viscosity solution of problem 2.2 converges uniformly (as $\varepsilon \rightarrow 0)$ on $\mathbb{R}^{N} \times[0, T)$ to the viscosity solution of the Hamilton-Jacobi initial value problem

$$
\begin{cases}\frac{\partial u}{\partial t}+\bar{F}(\nabla u)=0 & \text { in } \mathbb{R}^{N} \times[0, T), \\ u(x, 0)=u_{0}(x) & \text { in } \mathbb{R}^{N}\end{cases}
$$

where the Hamiltonian $\bar{F}(p)$ is uniquely determined by the requirement that there exists a periodic viscosity solution $v$ to the following degenerate elliptic equation:

$$
F\left(\nabla^{2} v(y), \nabla v(y)+p, y\right)=\bar{F}(p) .
$$

(We call the relation (2.6) the cell problem.)

Proof. Consider the approximating problem

$$
\delta w^{\delta}(y)+F\left(\nabla_{y}^{2} w^{\delta}(y), \nabla_{y} w^{\delta}(y)+p, y\right)=0,
$$

for some $p \in \mathbb{R}^{N}$ and some $\delta \in(0,1)$. This problem has a unique continuous viscosity solution $w^{\delta}$ (see [13]). Moreover, the periodicity of $F$ and the uniqueness of the solution $w^{\delta}$ imply that $w^{\delta}$ has to be periodic with the same unit cell $Y_{N}=[0,1]^{N}$. 
At any point where $w^{\delta}$ attains its maximum, its Hessian $\nabla_{y}^{2} w^{\delta}$ is negative semidefinite, so the degenerate ellipticity of $F$ implies that

$$
\delta w^{\delta}(y)=-F\left(\nabla_{y}^{2} w^{\delta}(y), \nabla_{y} w^{\delta}(y)+p, y\right) \leqslant F(0, p, y) \leqslant\|F(0, p, \cdot)\|_{L^{\infty}\left(Y_{N}\right)}
$$

whenever $y$ is a maximum of $w^{\delta}$. In a similar manner it can be argued that

$$
\delta w^{\delta}(y) \geqslant-\|F(0, p, \cdot)\|_{L^{\infty}\left(Y_{N}\right)}
$$

whenever $w^{\delta}$ reaches its minimum. It follows that

$$
\sup _{0<\delta<1}\left\|\delta w^{\delta}\right\|_{L^{\infty}\left(Y_{N}\right)} \leqslant\|F(0, p, \cdot)\|_{L^{\infty}\left(Y_{N}\right)} .
$$

Using this and the assumption on the Hölder continuity of the solution of 2.4), we conclude that there exists a subsequence $\delta_{j} \rightarrow 0$ such that $v^{\delta_{j}} \rightarrow v$ uniformly in $\mathbb{R}^{N}$ and $\delta_{j} w^{\delta_{j}} \rightarrow-\lambda$ uniformly in $\mathbb{R}^{N}$, where the function $v^{\delta}$ is defined by $v^{\delta}(y)=w^{\delta}(y)-\min _{Y_{N}} w^{\delta}$ and $\lambda$ is some constant. Thus we can pass to the limit $\delta \rightarrow 0$ in 2.7 to get

$$
F\left(\nabla_{y}^{2} v(y), D_{y} v(y)+p, y\right)=\lambda .
$$

We now want to prove the uniqueness of the constant $\lambda$ for which a function $v$ that satisfies $(2.8)$ exists. Assume by contradiction that there exists a second pair $(\tilde{v}, \tilde{\lambda})$ that satisfies $(2.8)$, with $\tilde{v}$ a periodic function and $\tilde{\lambda}>\lambda$. By adding a constant if necessary, we may also assume that $v>\tilde{v}$. Then, for some $\varepsilon$ small enough, we have

$$
\varepsilon \tilde{v}+F\left(\nabla_{y}^{2} \tilde{v}(y), D_{y} \tilde{v}(y)+p, y\right)>\theta>\varepsilon v+F\left(D_{y}^{2} v(y), D_{y} v(y)+p, y\right)
$$

for some constant $\theta$. Thus $\tilde{v}$ is a periodic supersolution of the equation

$$
F\left(\nabla_{y}^{2} v(y), D_{y} v(y)+p, y\right)+\varepsilon v-\theta=0
$$

and $v$ is a periodic subsolution of the same equation. Using the comparison principle for periodic solutions of geometric degenerate elliptic equations (see [13]), we arrive at $\tilde{v}>v$, in contradiction with our assumption that $v>\tilde{v}$. Thus the constant $\lambda$ for which a periodic solution $v$ to 2.8 exists is unique and we may denote it by $\bar{F}(p)$. All we have now left to prove is that the viscosity solution of problem 2.2 converges uniformly (as $\varepsilon \rightarrow 0$ ) on $\mathbb{R}^{N} \times[0, T)$ to the viscosity solution of the Hamilton-Jacobi initial value problem 2.5.

Since $F$ is degenerate elliptic and parabolic, problem 2.2 has a unique continuous viscosity solution $u^{\varepsilon}$ of on $\mathbb{R}^{N} \times[0, T]$ (Theorem 1.1 in [3], Theorem 4.3.5 in [18]). Let us define the function $u^{*}: \mathbb{R}^{N} \times[0, T) \rightarrow \mathbb{R}$ by

$$
u^{*}(x, t)=\limsup _{\varepsilon \rightarrow 0, z \rightarrow x, s \rightarrow t} u^{\varepsilon}(z, s) .
$$

We claim that

$$
\frac{\partial u^{*}}{\partial t}(x, t)+\bar{F}\left(\nabla u^{*}(x, t)\right) \leqslant 0
$$

in viscosity sense. By Remark 2.1.6 in [18], this is equivalent to the claim that $[2.9]$ holds in $\mathcal{F}$ viscosity sense (as defined in Definition 2.1.5 of [18]). 
To prove that 2.9 holds in $\mathcal{F}$-viscosity sense, consider a function $\phi \in C^{\infty}\left(\mathbb{R}^{N} \times[0, T)\right)$ such that $u^{*}-\phi$ has a strict local maximum at the point $\left(x_{0}, t_{0}\right)$, with

$$
u^{*}\left(x_{0}, t_{0}\right)=\phi\left(x_{0}, t_{0}\right) .
$$

We shall assume by contradiction that

$$
\frac{\partial \phi}{\partial t}+\bar{F}(\nabla \phi)=\theta \quad \text { at }\left(x_{0}, t_{0}\right),
$$

where $\theta$ is some positive constant. By the definition of $\bar{F}$, there exists a $Y$-periodic viscosity solution $v$ to the problem

$$
F\left(\nabla_{y}^{2} v(y), \nabla v(y)+\nabla_{x} \phi\left(x_{0}, t_{0}\right), y\right)=\bar{F}\left(\nabla_{x} \phi\left(x_{0}, t_{0}\right)\right) .
$$

Define the function $\phi^{\varepsilon}$ by

$$
\phi^{\varepsilon}(x, t)=\phi(x, t)+\varepsilon v\left(\frac{x}{\varepsilon}\right) .
$$

We claim that, if $\varepsilon$ is small enough, then

$$
\frac{\partial \phi}{\partial t}\left(x, t_{0}\right)+F\left(\varepsilon \nabla^{2} \phi^{\varepsilon}\left(x, t_{0}\right), \nabla \phi^{\varepsilon}\left(x, t_{0}\right), \frac{x}{\varepsilon}\right) \geqslant \frac{\theta}{2}
$$

in viscosity sense, in some ball $B\left(x_{0}, r\right)$ centered at $x_{0}$ with small enough radius $r$. To prove this, fix some $\psi \in C^{\infty}\left(\mathbb{R}^{N} \times[0, T)\right)$ such that $\phi^{\varepsilon}-\psi$ has a minimum at $\left(x_{1}, t_{0}\right) \in B\left(x_{0}, r\right) \times t_{0}$ with

$$
\phi^{\varepsilon}\left(x_{1}, t_{0}\right)=\psi\left(x_{1}, t_{0}\right) .
$$

Then the mapping $y \mapsto v(y)-\eta(y)$ has a minimum at $y_{1}=x_{1} / \varepsilon$, where

$$
\eta(y)=\frac{1}{\varepsilon}\left(\psi\left(\varepsilon y, t_{0}\right)-\phi\left(\varepsilon y, t_{0}\right)\right) .
$$

Since $v$ is a viscosity solution of 2.12 , this implies that

$$
F\left(\nabla_{y}^{2} \eta(y), \nabla_{y} \eta(y)+\nabla_{x} \phi\left(x_{0}, t_{0}\right), y\right) \geqslant \bar{F}\left(\nabla_{x} \phi\left(x_{0}, t_{0}\right)\right) .
$$

Thus, using 2.11,

$$
\frac{\partial \phi}{\partial t}\left(x_{0}, t_{0}\right)+F\left(\varepsilon \nabla^{2} \psi\left(x_{1}, t_{0}\right)-\varepsilon \nabla^{2} \phi\left(x_{1}, t_{0}\right), \nabla \psi\left(x_{1}, t_{0}\right)-\nabla \phi\left(x_{1}, t_{0}\right)+\nabla \phi\left(x_{0}, t_{0}\right), \frac{x_{1}}{\varepsilon}\right) \geqslant \theta .
$$

Wherever $\nabla \psi$ is not null, we can use the Lipschitz continuity of $F$ and the fact that

$$
\frac{\partial \phi}{\partial t}\left(x_{1}, t_{0}\right)=\frac{\partial \phi^{\varepsilon}}{\partial t}\left(x_{1}, t_{0}\right)=\frac{\partial \psi}{\partial t}\left(x_{1}, t_{0}\right),
$$

to infer that

$$
\frac{\partial \psi}{\partial t}+F\left(\varepsilon \nabla^{2} \psi, \nabla \psi, \frac{x_{1}}{\varepsilon}\right) \geqslant \frac{\theta}{2} \quad \text { at }\left(x_{1}, t_{0}\right)
$$

provided $\varepsilon$ and $r$ are small enough. 
At the points where $\nabla \psi$ is null, according to the definition of $\mathcal{F}$-viscosity, we can assume that the test function $\psi$ also satisfies $\nabla^{2} \psi=0$ so we can still pass to the limit using 2.3 .

Since the choice of the test function $\psi \in C^{\infty}\left(\mathbb{R}^{N} \times[0, T)\right)$ was arbitrary, the argument above establishes (2.13).

Since $\phi \in C^{\infty}\left(\mathbb{R}^{N} \times[0, T)\right)$, inequality 2.13 implies that

$$
\frac{\partial \phi}{\partial t}(x, t)+F\left(\varepsilon \nabla^{2} \phi^{\varepsilon}(x, t), \nabla \phi^{\varepsilon}(x, t), \frac{x}{\varepsilon}\right) \geqslant \frac{\theta}{4} \quad \text { in } B\left(x_{0}, r\right) \times\left[t_{0}-r^{\prime}, t_{0}+r^{\prime}\right]
$$

for some $r^{\prime}$ small enough. But $u^{\varepsilon}$ is a viscosity solution of 2.2), so the comparison principle for initial and boundary value problems for geometric degenerate parabolic equations on bounded domains (Theorem 3.6.1 in [18], Theorem 1.3 in [3]) yields

$$
\max _{B\left(x_{0}, r\right) \times B\left(t_{0}, r^{\prime}\right)}\left(u^{\varepsilon}-\phi^{\varepsilon}\right) \leqslant \max _{\partial\left(B\left(x_{0}, r\right) \times B\left(t_{0}, r^{\prime}\right)\right)}\left(u^{\varepsilon}-\phi^{\varepsilon}\right) .
$$

In the limit $\varepsilon \rightarrow 0$ this inequality becomes

$$
\left(u^{*}-\phi^{\varepsilon}\right)\left(x_{0}, t_{0}\right) \leqslant \max _{\partial\left(B\left(x_{0}, r\right) \times B\left(t_{0}, r^{\prime}\right)\right)}\left(u^{*}-\phi^{\varepsilon}\right),
$$

which contradicts 2.10). This establishes 2.97.

Also, by the continuity of $u^{\varepsilon}$ and the definition of $u^{*}$, the initial condition in 2.2 implies

$$
u^{*}\left(x_{0}, 0\right)=u_{0}(x) \text { in } \mathbb{R}^{N} .
$$

Then the comparison principle for 2.5 , along with 2.9$]$ and 2.14 , gives

$$
u^{*} \leqslant u \text {. }
$$

Similarly it can be proved that

$$
u \leqslant u_{*}=\liminf _{\varepsilon \rightarrow 0, z \rightarrow x, s \rightarrow t} u^{\varepsilon}(s, z)
$$

and since we obviously have $u_{*} \leqslant u^{*}$, we conclude that $u=u^{*}=u_{*}$.

Hence $u^{\varepsilon}$ converges uniformly (as $\varepsilon \rightarrow 0$ ) to $u$, the solution of 2.5.

REMARK 2 In stating Theorem 1, we assumed that the viscosity solution of the problem 2.4 is Hölder continuous in $x$ and its Hölder coefficient is bounded with respect to $\delta$ for $\delta \in(0,1)$. This assumption is stronger than the established continuity of the viscosity solutions of [2.4] (see [13]). However the assumption does appear plausible in light of the regularity theory for free boundary problems [6, 7, 8]. In any case, we point out that this assumption of uniform Hölder continuity is used only for proving existence of the solution of the cell problem (2.8). So an alternative approach to stating the theorem would be to assume that the solution of 2.8) exists. Again, this assumption is beyond what is known in general. However, we show the existence of solution of the cell problem for a special class of functions $F$ and $f$ (i.e., for curvature-sensitive evolution with a special class of defect microstructure) in Section 3.1. Our numerical computations in Section 3.2 and discussion in Section 3.3 also support existence in other examples.

We now show that certain properties of the operator $F$ are inherited by the limit Hamiltonian $\bar{F}$. 
THEOREM 3 If the operator $F$ in $(2.2)$ satisfies

$$
\lim _{|p| \rightarrow \infty} F(0, p, y)=\infty \quad \text { uniformly in } y,
$$

then the same holds for the homogenized Hamiltonian $\bar{F}$ :

$$
\lim _{|p| \rightarrow \infty} \bar{F}(p)=\infty
$$

Proof. We refer to the approximating problem (2.7) in the proof of Theorem 1. Writing (2.7) at point $y_{0}$ where $w^{\delta}$ attains its maximum we get

$$
\delta w^{\delta}\left(y_{0}\right)+F\left(0, p, y_{0}\right) \leqslant 0 .
$$

For any $M>0$, if $p$ is large enough, 2.15) implies that

$$
-\delta w^{\delta} \geqslant M
$$

But $-\delta w^{\delta} \rightarrow \bar{F}(p)$ uniformly as $\delta \rightarrow 0$, so the inequality above proves the theorem.

THEOREM 4 If the operator $F$ in 2.2 is convex with respect to its first two variables on $S_{N} \times$ $\mathbb{R}^{N} \backslash\{0\}$, then the homogenized Hamiltonian $\bar{F}$ is convex in $p$.

Proof. Fix $p, q \in \mathbb{R}^{N} \backslash\{0\}, x \in \mathbb{R}^{N}$ and let $v^{p}, v^{q}$ and $v^{(p+q) / 2}$ be the $Y_{N}$-periodic viscosity solutions of the following cell problems:

$$
\left\{\begin{array}{l}
F\left(\nabla^{2} v^{p}(y), \nabla v^{p}(y)+p, y\right)=\bar{F}(p), \\
F\left(\nabla^{2} v^{q}(y), \nabla v^{q}(y)+q, y\right)=\bar{F}(q), \\
F\left(\nabla^{2} v^{(p+q) / 2}(y), \nabla v^{(p+q) / 2}(y)+\frac{p+q}{2}, y\right)=\bar{F}\left(\frac{p+q}{2}\right) .
\end{array}\right.
$$

By subtracting a constant from $v^{(p+q) / 2}$ if necessary, we may also assume that

$$
v^{(p+q) / 2}<\frac{1}{2}\left(v^{p}+v^{q}\right) \quad \text { in } \mathbb{R}^{N} .
$$

To prove the convexity of $\bar{F}$, we assume by contradiction that

$$
\bar{F}\left(\frac{p+q}{2}\right)>\frac{1}{2}(\bar{F}(p)+\bar{F}(q)) .
$$

We claim that

$$
F\left(\nabla^{2} \frac{v^{p}+v^{q}}{2}(y), \nabla \frac{v^{p}+v^{q}}{2}(y)+\frac{p+q}{2}, y\right) \leqslant \frac{1}{2}(\bar{F}(p)+\bar{F}(q)) \quad \text { in } \mathbb{R}^{N} .
$$

To prove this, let $w=\frac{1}{2}\left(v^{p}+v^{q}\right)$ and $w_{\varepsilon}=\eta_{\varepsilon} * w$, where $\eta_{\varepsilon}$ is the mollifier with support in the ball $B(0, \varepsilon)$, i.e., an infinitely differentiable function $\eta_{\varepsilon} \geqslant 0$ such that

$$
\eta_{\varepsilon}(x)=\eta_{\varepsilon}(|x|), \quad \int_{0}^{\varepsilon} \eta_{\varepsilon}(x) x \mathrm{~d} x=1, \quad \eta_{\varepsilon}(x)=0 \text { if } x \geqslant \varepsilon .
$$


Then

$$
\begin{aligned}
F\left(\nabla^{2} w_{\varepsilon}(y), \nabla w_{\varepsilon}(y)+\frac{p+q}{2}, y\right) \leqslant & \int_{B(y, \varepsilon)} \eta_{\varepsilon}(y-z) F\left(\nabla^{2} w(z), \nabla w(z)+\frac{p+q}{2}, y\right) \mathrm{d} z \\
= & \int_{B(y, \varepsilon)} \eta_{\varepsilon}(y-z) F\left(\nabla^{2} w(z), \nabla w(z)+\frac{p+q}{2}, z\right) \mathrm{d} z+o(1) \\
\leqslant & \frac{1}{2} \int_{B(y, \varepsilon)} \eta_{\varepsilon}(y-z) F\left(\nabla^{2} v^{p}(z), \nabla v^{p}(z)+p, z\right) \mathrm{d} z \\
& +\frac{1}{2} \int_{B(y, \varepsilon)} \eta_{\varepsilon}(y-z) F\left(\nabla^{2} v^{q}(z), \nabla v^{q}(z)+q, z\right) \mathrm{d} z+o(1) \\
= & \frac{1}{2} \bar{F}(p)+\frac{1}{2} \bar{F}(q)+o(1)
\end{aligned}
$$

as $\varepsilon \rightarrow 0$. In the limit $\varepsilon=0$, we get 2.18 .

But 2.17) and 2.18 imply, by the comparison principle for periodic solutions of geometric degenerate elliptic equations, that

$$
\frac{1}{2}\left(v^{p}+v^{q}\right) \leqslant v^{(p+q) / 2}
$$

which contradicts 2.16.

We now specialize to $F$ defined by the interface propagation problem (2.1). Theorem 1 tells us that its viscosity solution converges uniformly (as $\varepsilon \rightarrow 0)$ on $\mathbb{R}^{N} \times[0, T)$ to the viscosity solution of the Hamilton-Jacobi initial value problem $\sqrt{2.5}$, with $\bar{F}(p)$ uniquely determined by the requirement that there exists a periodic viscosity solution $v$ to the following cell problem:

$$
f(x)|\nabla v+p|-c\left(\delta_{i j}-\frac{\left(v_{x_{i}}+p_{i}\right)\left(v_{x_{j}}+p_{j}\right)}{|\nabla v+p|^{2}}\right) v_{x_{i} x_{j}}=\bar{F}(p) .
$$

Proposition 5 For $F$ defined by 2.1 we have

$$
\bar{F}(p)=\bar{f}\left(\frac{p}{|p|}\right)|p| .
$$

Proof. If $v$ is a viscosity solution of 2.19 corresponding to $p$ and $\bar{F}(p)$, then it is easily verified that $\alpha v$ is a viscosity solution of 2.19 corresponding to $\alpha p$ and $\bar{F}(\alpha p)$. It follows then from the uniqueness of $\bar{F}$ that $\bar{F}(\alpha p)=\alpha \bar{F}(p)$, and we obtain the desired result.

We conclude that the effective motion of a curvature driven interface through a heterogeneous medium is an anisotropic geometric one, similar to the effective motion studied in [11].

\section{Examples}

\subsection{Laminates}

Assume that the function $f(x)$ takes two values $f_{1}$ and $f_{2}$ in alternating stripes with volume fractions $\mu$ and $1-\mu$ respectively, as shown in Figure 3.1 . Specifically, let

$$
f\left(x_{1}, x_{2}\right)= \begin{cases}f_{1} & \text { if } x_{1} \in[0, \mu / 2] \cup[1-\mu / 2,1], \\ f_{2} & \text { if } x_{1} \in(\mu / 2,1-\mu / 2)\end{cases}
$$




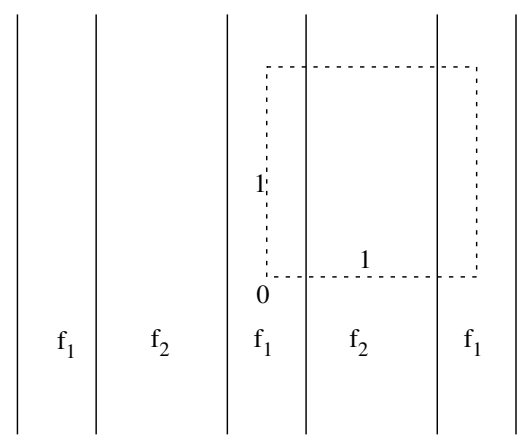

FIG. 3.1. Laminated material.

with $f$ extended by periodicity outside the strip $x_{1} \in[0,1]$. We assume that $f_{1}>f_{2}$ without loss of generality.

We study the propagation of an interface whose average normal is in the $x_{2}$ direction. We claim that if we find a self-similar front $\mathcal{F},[0,1]$-periodic in $x_{1}$, that propagates by uniform translation in the $x_{2}$ direction, then the translation velocity will be the effective normal velocity in this upwards $x_{2}$ direction. Indeed, any other front that has effective normal parallel to $x_{2}$ can be initially trapped between two copies of $\mathcal{F}$ and remains trapped between them for all time by the comparison principle. It follows that its effective velocity has to be the same as the effective velocity of $\mathcal{F}$.

We begin by trying to find a self-similar front $\mathcal{F}$ that can also be written as the graph $x_{2}=g\left(x_{1}\right)$ of a $[0,1]$-periodic function $g$. If $v$ denotes the constant upwards-translation velocity of $\mathcal{F}$, then its normal velocity and curvature at any point are

$$
v_{n}=(0, v) \cdot n=\frac{v}{\sqrt{1+g^{\prime}\left(x_{1}\right)^{2}}}, \quad \kappa=-\frac{g^{\prime \prime}\left(x_{1}\right)}{\left(\sqrt{1+g^{\prime}\left(x_{1}\right)^{2}}\right)^{3}} .
$$

Plugging this in our normal velocity formula 1.1 , we get

$$
\frac{v}{\sqrt{1+z\left(x_{1}\right)^{2}}}=f\left(x_{1}\right)+c \frac{z^{\prime}\left(x_{1}\right)}{\left(\sqrt{1+z\left(x_{1}\right)^{2}}\right)^{3}},
$$

where $z\left(x_{1}\right)=g^{\prime}\left(x_{1}\right)$. This equation can also be written as

$$
v \cos u\left(x_{1}\right)=f\left(x_{1}\right)+c u^{\prime}\left(x_{1}\right) \cos u\left(x_{1}\right),
$$

in the new unknown function $u\left(x_{1}\right)=\arctan z\left(x_{1}\right)$. The variables in this ordinary differential equation can be separated

$$
\frac{\mathrm{d} u \cos u}{v \cos u-f}=\frac{\mathrm{d} x_{1}}{c}
$$

in all the regions where $f$ is constant.

Due to the periodicity of $f$ and $g$ and due to the symmetry of $f$ with respect to the axis $x_{1}=1 / 2$, the front $\mathcal{F}$ has critical points at $x_{1}=0$ and $x_{1}=1 / 2$. This means that

$$
u(0)=u(1 / 2)=0 \text {. }
$$


We can obtain two conditions for $\bar{u}=u(\mu / 2)$, by integrating (3.2) with starting points $x_{1}=0$ (forward) and $x_{1}=1 / 2$ (backward), respectively:

$$
\begin{aligned}
\frac{\mu}{2 c} & =-\int_{0}^{\bar{u}} \frac{\mathrm{d} u \cos u}{v \cos u-f_{1}}, \\
\frac{1-\mu}{2 c} & =\int_{0}^{\bar{u}} \frac{\mathrm{d} u \cos u}{v \cos u-f_{2}} .
\end{aligned}
$$

We now show that this pair of equations can be solved simultaneously for the two unknowns $\bar{u} \in$ $\left[0, u_{\max }\right]$ and $v \in\left[f_{2}, f_{1}\right]$ if $c \leqslant \mu / 2 k$ where

$$
u_{\max }=\arccos \left(\frac{f_{2}}{f_{1}}\right) \in[0, \pi / 2], \quad k=\int_{0}^{u_{\max }} \frac{\mathrm{d} u \cos u}{f_{1}-f_{2} \cos u} .
$$

Note $0 \leqslant k<\infty$.

First, given any $v \in\left[f_{2}, f_{1}\right]$, we show that $(3.5)$ has a solution $\bar{u} \in\left[0, u_{\max }\right]$, and further that this solution $\bar{u}(v)$ is decreasing. Let $u^{*}=\arccos \left(f_{2} / v\right) \in\left[0, u_{\max }\right]$, and let

$$
g(\hat{u})=\int_{0}^{\hat{u}} \frac{\mathrm{d} u \cos u}{v \cos u-f_{2}} .
$$

Note that $g$ is increasing on $\left[0, u^{*}\right]$ and that

$$
\lim _{\hat{u} \rightarrow 0} g(\hat{u})=0, \quad \lim _{\hat{u} \rightarrow \infty} g(\hat{u})=\infty .
$$

Therefore, $g(\bar{u})=(1-\mu) / 2$ or 3.5 has a solution in $\left[0, u^{*}\right] \subset\left[0, u_{\max }\right]$. Further, it is clear from the right hand side of 3.5 that this solution is increasing.

Second, given any $\bar{u} \in\left[0, u_{\max }\right]$, we show that (3.4] has a solution $v=\bar{v} \in\left[f_{2}, f_{1}\right]$, and that $\bar{v}(\bar{u})$ is decreasing. Let

$$
h(\hat{v})=\int_{0}^{\bar{u}} \frac{\mathrm{d} u \cos u}{f_{1}-\hat{v} \cos u} .
$$

Note that $h$ is increasing on $\left[f_{2}, f_{1}\right]$, and that

$$
\lim _{\hat{v} \rightarrow f_{2}} h(\hat{v})=\int_{0}^{\bar{u}} \frac{\mathrm{d} u \cos u}{f_{1}-f_{2} \cos u}<k \leqslant \frac{\mu}{2 c}, \quad \lim _{\hat{v} \rightarrow f_{1}} h(\hat{v})=\infty .
$$

Therefore, $h(\hat{v})=\mu / 2 c$ or 3.4 has a solution in $\left[f_{2}, f_{1}\right]$. Further, it is clear from the right hand side of (3.4) that this solution is decreasing.

Now define $\phi(v)=\bar{v}(\bar{u}(v))$. This mapping is decreasing from $\left[f_{2}, f_{1}\right]$ to itself, so it must have a fixed point $v$. Then $\{v, \bar{u}(v)\}$ are the simultaneous solutions of (3.4) and (3.5) that we seek.

This result can be extended for $c$ larger than $\mu / 2 k$. Note from 3.5 that $\bar{u}$ becomes smaller with $c$, and we can use this to improve the lower estimate in (3.6). The details are cumbersome and omitted since we will deal with the large $c$ case in some generality later.

Figure 3.2 shows the dependence $v=v(c)$ for fixed values of $f_{1}(=1)$ and $\mu(=1 / 2)$ and for various $f_{2}$ varying from -.05 to .95 (the uppermost graph is for the highest value of the parameter $\left.f_{2}\right)$. For each value of $f_{2}, v$ decreases with $c$, reaches the limit $\max \left(f_{1}, f_{2}\right)=1$ as $c \rightarrow 0$ and 


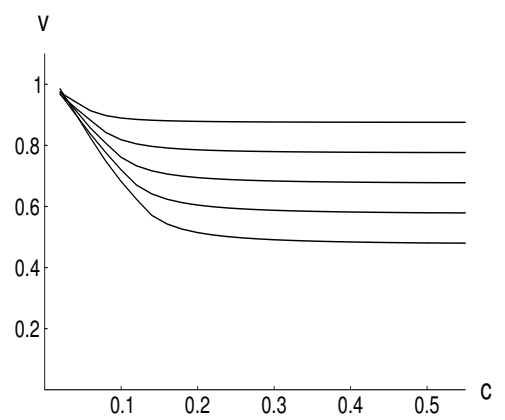

FIG. 3.2. The effective normal velocity $v$ in the $e_{2}$ direction for a laminate with $f_{1}=1, \mu=0.5$ as a function of $c$ for various values of $f_{2}$ from -0.05 (lowest curve) to 0.95 (highest curve).

the average $\frac{1}{2}\left(f_{1}+f_{2}\right)$ as $c \rightarrow \infty$. We note that the former limit is consistent with the case $c=0$ (Example 1 in Section 4 of [11]) and we now discuss the latter limit.

Combining (3.4) and 3.5, we obtain

$$
-\frac{1}{\mu} \int_{0}^{\bar{u}} \frac{\mathrm{d} u \cos u}{v \cos u-f_{1}}=\frac{1}{1-\mu} \int_{0}^{\bar{u}} \frac{\mathrm{d} u \cos u}{v \cos u-f_{2}} .
$$

Applying the mean value theorem to each integral, we find $u_{1}, u_{2} \in[0, \bar{u}]$ such that

$$
-\frac{1}{\mu}\left(\frac{\cos u_{1}}{v \cos u_{1}-f_{1}}\right)=\frac{1}{1-\mu}\left(\frac{\cos u_{2}}{v \cos u_{2}-f_{2}}\right) .
$$

Now, it is clear from (3.4) and 3.5 that $\bar{u}$, and consequently $u_{1}, u_{2}$, decreases to zero as $c$ goes to infinity. Therefore, up to $o(1 / c)$, the equation above becomes

$$
-\frac{1}{\mu\left(v-f_{1}\right)}=\frac{1}{(1-\mu)\left(v-f_{2}\right)},
$$

and this in turn implies that

$$
v=\mu f_{1}+(1-\mu) f_{2}=\int_{0}^{1} f\left(x_{1}\right) \mathrm{d} x_{1}
$$

as suggested by Figure 3.2. We shall return to this limit in the general case in Section 4.

The self-similar fronts not only allow us to calculate the values of the effective normal velocity but they also seem to act as attractors in the space of solutions for (1.2), as is clear from the following numerical simulation on a $64 \times 64$ grid. We use a semi-implicit scheme when discretizing (1.2):

$$
\frac{h^{n+1}-h^{n}}{\Delta t}=f P+c\left|\nabla h^{n}\right| \operatorname{div} \frac{\nabla h^{n+1}}{\left|\nabla h^{n}\right|},
$$

where

$$
P= \begin{cases}\sqrt{\max \left(p_{-}^{x}, 0\right)^{2}+\min \left(p_{+}^{x}, 0\right)^{2}+\max \left(p_{-}^{y}, 0\right)^{2}+\min \left(p_{+}^{y}, 0\right)^{2}} & \text { if } f>0, \\ \sqrt{\min \left(p_{-}^{x}, 0\right)^{2}+\max \left(p_{+}^{x}, 0\right)^{2}+\min \left(p_{-}^{y}, 0\right)^{2}+\max \left(p_{+}^{y}, 0\right)^{2}} & \text { if } f \leqslant 0,\end{cases}
$$




$$
\begin{aligned}
& p_{-}^{x}=\frac{1}{\Delta x} D_{x}^{-} \phi_{i, j}^{n}+\frac{1}{2 \Delta x} \operatorname{minmod}\left(D_{x}^{-} D_{x}^{+} \phi_{i, j}^{n}, D_{x}^{-} D_{x}^{+} \phi_{i-1, j}^{n}\right), \\
& p_{+}^{x}=\frac{1}{\Delta x} D_{x}^{-} \phi_{i+1, j}^{n}+\frac{1}{2 \Delta x} \operatorname{minmod}\left(D_{x}^{-} D_{x}^{+} \phi_{i+1, j}^{n}, D_{x}^{-} D_{x}^{+} \phi_{i, j}^{n}\right), \\
& p_{-}^{y}=\frac{1}{\Delta x} D_{y}^{-} \phi_{i, j}^{n}+\frac{1}{2 \Delta x} \operatorname{minmod}\left(D_{y}^{-} D_{y}^{+} \phi_{i, j}^{n}, D_{y}^{-} D_{y}^{+} \phi_{i, j-1}^{n}\right) \text {, } \\
& p_{+}^{y}=\frac{1}{\Delta x} D_{y}^{-} \phi_{i, j+1}^{n}+\frac{1}{2 \Delta x} \operatorname{minmod}\left(D_{y}^{-} D_{y}^{+} \phi_{i, j+1}^{n}, D_{y}^{-} D_{y}^{+} \phi_{i, j}^{n}\right) \text {, } \\
& \operatorname{minmod}(u, v)= \begin{cases}\operatorname{sign}(u) \min (|u|,|v|) & \text { if } u v>0, \\
0 & \text { if } u v \leqslant 0,\end{cases} \\
& D_{x}^{-} \phi_{i, j}=\phi_{i, j}-\phi_{i-1, j} \quad \text { (backward), } \\
& D_{x}^{+} \phi_{i, j}=\phi_{i+1, j}-\phi_{i, j} \quad \text { (forward). }
\end{aligned}
$$

For the rest of the spatial derivatives, we simply use centered difference operators. We solve the resulting linear system by means of a conjugate gradient method. Since we wish to avoid large gradients in the level set function, we keep it close to the signed distance function to the interface by using, after each time step, a reinitialization procedure developed by Sussman, Smereka and Osher in [31].

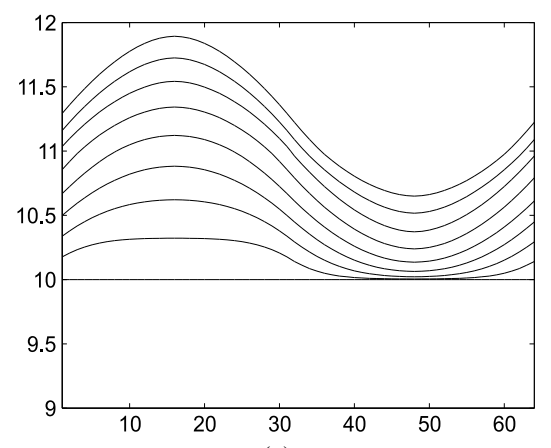

(a)

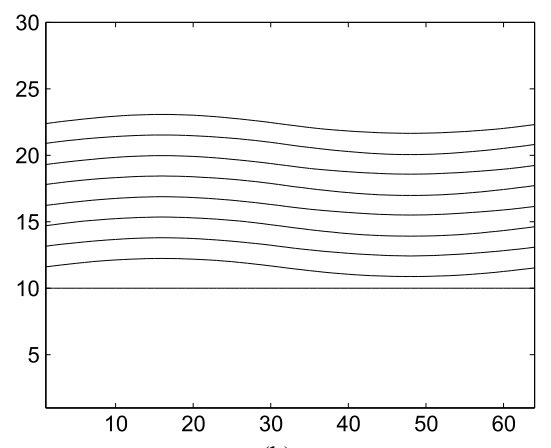

(b)

FIG. 3.3. Snapshots of an initially flat interface in a laminate with parameters 3.7) at (a) every 50 time steps and (b) every 500 time steps.

Figure 3.3 a) shows several snapshots of an initially flat front moving in the laminate with the parameters

$$
\mu=1 / 2, \quad f_{1}=1, \quad f_{2}=0, \quad c=1,
$$

taken at every 50 time steps (we used the time step $\Delta t=0.0001$ ). We see how this initially flat interface rapidly approaches the self-similar shape. It subsequently starts a uniform upward translation. This is shown in Figure 3.3 b), which shows snapshots of the same simulation taken at every 500 time steps.

Figure 3.4 shows the evolution of an initially flat interface for the laminate with the parameters

$$
\mu=1 / 2, \quad f_{1}=1, \quad f_{2}=-0.6, \quad c=4 .
$$

The front initially moves downwards in the part where $f$ is negative but is subsequently dragged upwards by the other regions and eventually assumes the self-similar shape that translates upwards. 


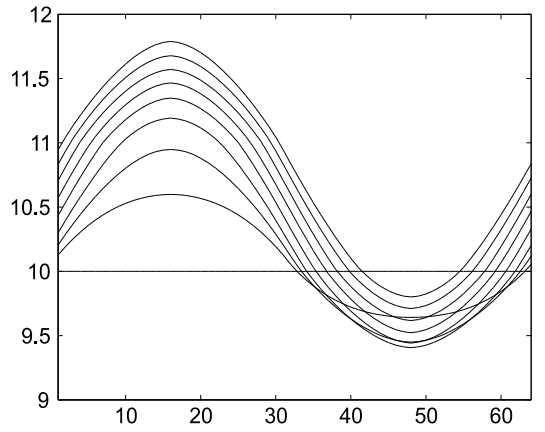

(a)

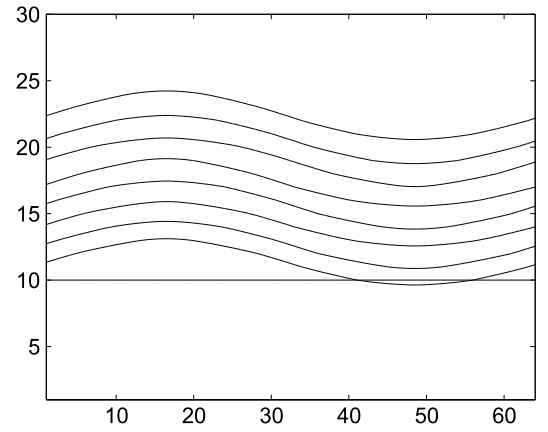

(b)

FIG. 3.4. Snapshots of an initially flat interface in a laminate with parameters 3.8 where $f$ takes both positive and negative values at (a) every 100 time steps and (b) every 2000 time steps.

In both these cases, the effective normal velocity (which can be calculated from the distance between the level sets) agrees with that obtained earlier.

\subsection{Other geometries}

For mediums which do not have a laminate-type heterogeneity, self-similar interfaces do not generally exist. However, Theorem 1 suggests the existence of interfaces which are self-similar to the extent that they assume the same shape after they have propagated over one unit cell of $f$. In certain situations, these self-similar interfaces also seem to act as attractors since any initial interface approaches them as time evolves.

As an example, consider

$$
f=10+5 \sin \frac{2 \pi x}{64} \sin \frac{2 \pi y}{64} .
$$

Figure 3.5 shows the evolution of an initially flat interface positioned at the height $y=10$ with a $64 \times 64$ unit cell. The computational grid is also $64 \times 64$. The snapshots are taken at every 100 time steps (with $\Delta t=0.0001$ ). In the lower half of the unit square, the left part is faster than the right

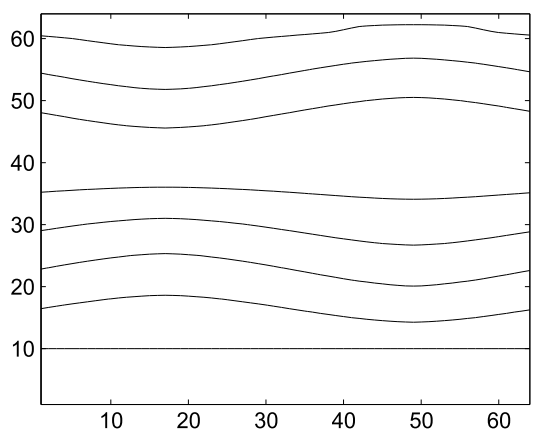

FIG. 3.5. Motion of a curvature driven interface in a medium with a sinusoidal normal velocity law. The figure shows snapshots of an initially flat interface at every 100 time steps. 
so the front assumes a shape similar to the one observed in the laminate examples. However, as the interface approaches the height $j=32$, the interface starts to flatten. In the upper half of the unit square, the situation is reversed: the right part of the interface will be faster than the left and then it again starts to flatten as it approaches the upper boundary of the unit cell.

\subsection{Trapping}

Of particular interest are the interfaces that are trapped, i.e., interfaces which evolve to a stationary position. We begin with a laminate and seek conditions when one has a stationary interface with average normal in the $x_{2}$ direction. We assume, as before, that the interface is a graph. Proceeding as before (or setting $v=0$ in 3.2 ), we obtain

$$
\mathrm{d} u \cos u=-\frac{f}{c} \mathrm{~d} x
$$

subject to 3.3 . If

$$
c>\max \left\{\frac{\left|f_{1}\right| \mu}{2}, \frac{\left|f_{2}\right|(1-\mu)}{2}\right\},
$$

then we can solve this explicitly in regions where $f$ is constant to obtain

$$
u\left(x_{1}\right)= \begin{cases}\arcsin \frac{-f_{1} x_{1}}{c} & \text { for } x_{1} \in[0, \mu / 2], \\ \arcsin \frac{f_{2}\left(1 / 2-x_{1}\right)}{c} & \text { for } x_{1} \in[\mu / 2,1 / 2] .\end{cases}
$$

The continuity of $u$ at $x_{1}=\mu / 2$ gives

$$
\arcsin \frac{-f_{1} \mu}{2 c}=\arcsin \frac{f_{2}(1-\mu)}{2 c},
$$

which implies

$$
\int_{0}^{1} f\left(x_{1}\right) \mathrm{d} x_{1}=\mu f_{1}+(1-\mu) f_{2}=0 .
$$

Thus we can construct a stationary front when the average of $f$ is zero and $c$ is large enough. We note that the converse is also true: if the average of $f$ is positive (resp. negative), then the average velocity is positive (resp. negative) for $c=\infty$ and therefore positive (resp. negative) for any $c \geqslant 0$ by the monotonicity of the effective velocity in $c$ in laminates.

Similar trapping conditions can also be deduced for other geometries. For example, consider the case of a two-dimensional medium with $f$ constant and positive except on a square array of circular inclusions where $f$ is a (different) negative constant. In particular, consider $f:[0,1]^{2} \rightarrow \mathbb{R}$ defined by

$$
f\left(x_{1}, x_{2}\right)= \begin{cases}f_{1} & \text { if }\left(x_{1}-1 / 2\right)^{2}+\left(x_{2}-1 / 2\right)^{2}>r^{2}, \\ f_{2} & \text { if }\left(x_{1}-1 / 2\right)^{2}+\left(x_{2}-1 / 2\right)^{2}<r^{2}\end{cases}
$$

with $f$ extended by periodicity outside the square $[0,1]^{2}$, where $r \in[0,1 / 2)$ and $f_{1}>0>f_{2}$ are constants. 


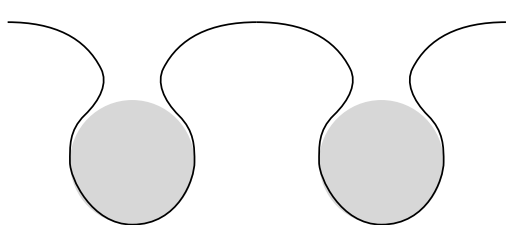

(a)

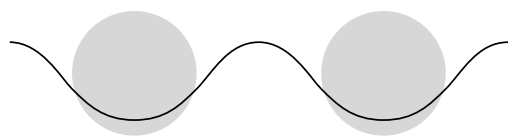

(b)

FIG. 3.6. An interface can propagate by (a) looping around a slow-velocity inclusion if the curvature coefficient is sufficiently small or (b) cutting through a slow-velocity inclusion if the curvature coefficient is sufficiently large.

Stationary fronts with overall normal $e_{2}$ can be constructed using the same method as in the laminate if

$$
f_{1} \leqslant \frac{c}{r} \leqslant-f_{2}
$$

Again, using the comparison principle, we conclude that no other front is able to break through these stationary barriers, so the effective normal velocity in the $e_{2}$ direction is null.

The trapping conditions in 3.10 are sharp in the following sense: If the first inequality is not satisfied, then the curvature is not penalized sufficiently and the front is able to propagate by looping around the slow-speed inclusions (see Figure 3.6(a)); if the second inequality is not satisfied, then the resistance to phase change inside the inclusion is not large enough and the curvature allows it to continue propagating by cutting through the inclusions (see Figure 3.6.(b)).

Finally, note from 3.10 that in this example, unlike in a laminate, the effective normal velocity is not monotone in $c$. The laminate is an exception in this regard and in general we do not expect monotonicity in $c$.

\section{The effective normal velocity for large $c$}

We saw in our study of laminates that one can obtain a simple characterization of the effective normal velocity when the curvature coefficient $c$ becomes very large. We now generalize this result to other microstructures. Indeed, we show that the average normal velocity of an interface propagating in the overall direction $p$ is given by

$$
V(p)=\bar{f}(p)=\left\langle\langle f\rangle_{p^{\perp}}^{-1}\right\rangle_{p}^{-1},
$$

where $\langle\cdot\rangle_{p^{\perp}}$ denotes the average of a $Y_{N}$-periodic function on an $N-1$-dimensional hyperplane that is orthogonal to $p$, and $\langle\cdot\rangle_{p}$ denotes the average of a periodic function of one variable in the direction parallel to $p$.

Heuristically, as $c$ becomes large, the curvature is severely penalized, so the interface evolves to become flat in the limit $c \rightarrow \infty$. The instantaneous normal velocity of this flat interface is given by the average of $f$ over the interface. We thus obtain a one-dimensional problem: a flat interface propagating normal to itself with a position-dependent velocity. Therefore, following Bhattacharya [5] (also see Abeyaratne, Chu and James [1]), the effective velocity is the harmonic mean of its instantaneous normal velocity. We thus obtain the characterization (4.1) above.

The rest of the section justifies this intuition. We prove the result in two dimensions, and justify it through formal asymptotic analysis in higher dimensions. As a prelude, we make the following observation. Since the effective normal velocity is a continuous function of direction (Theorem 3 ), 
it suffices to consider only 'rational' directions, i.e., directions where the ratios of components of the normal vector referred to a rectangular cartesian system aligned with the unit cell are rational. Then it is possible to choose a 'super unit cell', a cube which can be regarded as a unit cell of periodicity of $f$ and which has one edge aligned to the normal. By rescaling if necessary, we may then take without loss of generality the unit cell to be a unit cartesian cube and the overall direction of propagation to be $e_{N}$ parallel to $x_{N}$.

We begin with the two-dimensional case. We first prove that, for $c$ large enough, any sufficiently smooth interface in $\mathbb{R}^{2}$ with overall normal $e_{2}$ that is initially a graph remains so for all future time. Assume that the initial interface can be described as the graph $y=g(x)$ of a $[0,1]$-periodic function $g(\cdot, 0): \mathbb{R} \rightarrow \mathbb{R}$. As we have seen in the previous section (see 3.1), the equation of motion for the function $g$ is

$$
\frac{g_{t}(x, t)}{\sqrt{1+g_{x}(x, t)^{2}}}=f(x, g(x, t))+c \frac{g_{x x}(x, t)}{\left(\sqrt{1+g_{x}(x, t)^{2}}\right)^{3}}
$$

as long as the interface can be written as a graph. By scaling time $s=c t$ we can rewrite this as

$$
g_{s}=k \sqrt{1+g_{x}^{2}}
$$

where

$$
k(x, s)=\frac{f(x, g(x, s))}{c}-\kappa(x, g(x, s))=\frac{f(x, g(x, s))}{c}+\frac{g_{x x}}{\left(1+g_{x}^{2}\right)^{3 / 2}} .
$$

Differentiating 4.2 with respect to $x$, we get the following expression for $g_{x s}$ :

$$
g_{x s}=k_{x} \sqrt{1+g_{x}^{2}}+k \frac{g_{x} g_{x x}}{\sqrt{1+g_{x}^{2}}} .
$$

But, from 4.3),

$$
g_{x x}=\left(k-\frac{f}{c}\right)\left(1+g_{x}^{2}\right)^{3 / 2}
$$

Plugging this into 4.4 , we get

$$
g_{x s}=k_{x} \sqrt{1+g_{x}^{2}}+k\left(k-\frac{f}{c}\right) g_{x}\left(1+g_{x}^{2}\right) .
$$

Differentiating 4.6 with respect to $x$ and replacing all second order spatial derivatives $g_{x x}$ with the right hand side of 4.5 , we get the following expression for $g_{x x s}$ :

$$
\begin{aligned}
g_{x x s}= & k_{x x} \sqrt{1+g_{x}^{2}}+2 k_{x} g_{x}\left(k-\frac{f}{c}\right)\left(1+g_{x}^{2}\right) \\
& +k\left(k_{x}-\frac{f_{x}+f_{y} g_{x}}{c}\right) g_{x}\left(1+g_{x}^{2}\right)+k\left(k-\frac{f}{c}\right)^{2}\left(1+3 g_{x}^{2}\right)\left(1+g_{x}^{2}\right)^{3 / 2} .
\end{aligned}
$$

We now differentiate 4.3 with respect to $s$ and obtain

$$
k_{s}=\frac{f_{y} g_{s}}{c}+\frac{g_{x x s}}{\left(1+g_{x}^{2}\right)^{3 / 2}}-\frac{3}{2}\left(k-\frac{f}{c}\right) \frac{2 g_{x} g_{x s}}{1+g_{x}^{2}},
$$


which, after replacing $g_{x s}$ and $g_{x x s}$ with the expressions obtained in (4.6) and (4.7) and some algebraic manipulation, becomes

$$
k_{s}=\frac{k_{x x}}{\sqrt{1+g_{x}^{2}}}+\frac{f}{c} \frac{g_{x}}{\sqrt{1+g_{x}^{2}}} k_{x}+k\left(k-\frac{f}{c}\right)^{2}+k \frac{f_{y}-f_{x} g_{x}}{c \sqrt{1+g_{x}^{2}}} .
$$

We may choose the initial data for the interface to be such that

$$
k(x, g(x, 0)) \geqslant 0 .
$$

Then (4.8) enforces this inequality to stay valid at all times. Indeed, let $s_{1}$ be the first point in time when the function $k$ assumes the value 0 at some point $x_{1}$. If $x_{1}$ is an isolated zero point of $k$, then we have

$$
k_{x}\left(x_{1}, s_{1}\right)=0, \quad k_{x x}\left(x_{1}, t_{1}\right)>0
$$

and thus 4.8 gives $k_{s}\left(x_{1}, s_{1}\right)>0$. If $x_{1}$ is not an isolated zero point of $k$, then

$$
k_{x}\left(x_{1}, s_{1}\right)=k_{x x}\left(x_{1}, s_{1}\right)=0
$$

and thus (4.8) gives $k_{s}\left(x_{1}, s_{1}\right)=0$. It follows that, indeed, $k$ does not ever assume negative values.

By (4.3), the positivity of $k$ implies

$$
\frac{\mathrm{d}}{\mathrm{d} x} \frac{g_{x}}{\sqrt{1+g_{x}^{2}}} \geqslant-\frac{f(x, g)}{c},
$$

thus

$$
g_{x}^{2}\left(1-\left(\int_{x}^{x_{0}} \frac{f(x, g)}{c} \mathrm{~d} x\right)^{2}\right) \leqslant\left(\int_{x}^{x_{0}} \frac{f(x, g)}{c} \mathrm{~d} x\right)^{2}
$$

for all $x<x_{0}$, where $x_{0}$ is a point where $g$ assumes its maximum value. Moreover, a similar inequality will hold for $x>x_{0}$. Assuming that $c$ is large enough such that

$$
\left(\int_{x_{1}}^{x_{2}} \frac{f(x, g)}{c} \mathrm{~d} x\right)^{2} \leqslant 1
$$

for any $x_{1}$ and $x_{2}$, (4.9) and its analogue for values of $x$ with $x>x_{0}$ will provide finite bounds on $g_{x}$. These bounds ensure, in particular, that the interface will be such that it can be written as the graph of the function $g$ at all times.

It also follows that we are able to use the governing equation for $g$,

$$
g_{t}=f(x, g) \sqrt{1+g_{x}^{2}}+c \frac{g_{x x}}{1+g_{x}^{2}},
$$

at all times. Integrating this with respect to $x$ yields

$$
\left(\int_{x_{1}}^{x_{2}} g(x, t) \mathrm{d} x\right)_{t}=\int_{x_{1}}^{x_{2}} f(x, g(x, t)) \mathrm{d} x+\left.c \arctan g_{x}\right|_{x_{1}} ^{x_{2}} .
$$

If we choose $x_{1}$ and $x_{2}$ to be ends of an interval of periodicity for $g$, then the last term on the right hand side vanishes and we are left with

$$
\left(\int_{x_{1}}^{x_{2}} g(x, t) \mathrm{d} x\right)_{t}=\int_{x_{1}}^{x_{2}} f(x, g(x, t)) \mathrm{d} x,
$$


which says that, at any time, the instantaneous effective speed of the interface equals the average of the values of $f$ along the front.

We now estimate the effective normal velocity for the case when the constant interfacial energy $c$ is very large. If $c$ is large enough such that (4.10) holds, (4.9) implies that

$$
\left|g_{x}\right| \leqslant m=m(c)=\frac{\max |f|}{\sqrt{c^{2}-\max |f|}},
$$

and thus, for every $x_{0} \in[0,1]$,

$$
\left|g\left(x_{0}, t\right)-\bar{g}(t)\right| \leqslant m(c),
$$

where

$$
\bar{g}(t)=\int_{0}^{1} g(x, t) \mathrm{d} x .
$$

This expresses the fact that, as $c \rightarrow \infty$, curvature becomes more and more penalized so the front becomes flatter and flatter. Using (4.11), (4.12) and the Lipschitz property of $f$ yields

$$
\begin{aligned}
\left|\bar{g}_{t}-\int_{0}^{1} f(x, \bar{g}(x)) \mathrm{d} x\right| & =\left|\int_{0}^{1} f(x, g(x, t)) \mathrm{d} x-\int_{0}^{1} f(x, \bar{g}(x)) \mathrm{d} x\right| \\
& \leqslant \int_{0}^{1}|f(x, g(x, t))-f(x, \bar{g}(t))| \mathrm{d} x \\
& \leqslant \int_{0}^{1} L|g(x, t)-\bar{g}(t)| \mathrm{d} x \leqslant \int_{0}^{1} \operatorname{Lm}(c) \mathrm{d} x \leqslant \operatorname{Lm}(c) .
\end{aligned}
$$

Thus, at any instant of time, the velocity equals $\int_{0}^{1} f(x, \bar{g}(x)) \mathrm{d} x$, up to an error of order $o(1 / c)$. Then, as long as $\int_{0}^{1} f(x, y) \mathrm{d} x$ never vanishes, the speed with which the average front $\bar{g}(t)$ travels over one periodicity interval $[0,1]$ will be

$$
\left(\int_{0}^{1} \frac{1}{\int_{0}^{1} f(x, y) \mathrm{d} x} \mathrm{~d} y\right)^{-1}
$$

up to an error of order $o(1 / c)$. Since (4.12) ensures that the front $\mathcal{F}$ stays close to its average (given by $\bar{g}(t))$, (4.13) will also be the limit as $c \rightarrow \infty$ of the effective normal velocity of $\mathcal{F}$.

If the function $f$ is such that $\int_{0}^{1} f(x, y) \mathrm{d} x$ vanishes for some $y$, then the front will be trapped once it reaches that position and we have $V\left(e_{2}\right)=0$. We have proved the following.

PROPOSITION 6 The effective normal velocity of a periodic interface in $\mathbb{R}^{2}$ with overall normal $p$ and moving with the normal velocity law (1.1) has the limit

$$
V(p)=\bar{f}(p)=\left\langle\langle f\rangle_{p^{\perp}}^{-1}\right\rangle_{p}^{-1}
$$

as $c \rightarrow \infty$, as long as $\langle f\rangle_{p^{\perp}}$ never vanishes. If $\langle f\rangle_{p^{\perp}}$ vanishes at some point, then $\bar{f}(p)=0$.

We now extend this result to $\mathbb{R}^{N}$ through an asymptotic analysis of the unit cell problem 2.19. Accordingly, we look for a $Y_{N}$-periodic $v$ and constant $V$ such that

$$
f(x)\left|\nabla h+e_{N}\right|-c\left(\Delta h-\frac{\left\langle\left(e_{N}+\nabla h\right), \nabla^{2} h\left(e_{N}+\nabla h\right)\right\rangle}{\left|e_{N}+\nabla h\right|^{2}}\right)=V .
$$


Above we assume without loss of generality that $\left|e_{N}\right|=1$. We make the ansatz

$$
h(x)=u(x)+\frac{1}{c} v(x)+o\left(\frac{1}{c^{2}}\right), \quad V=c V_{-1}+V_{0}+o\left(\frac{1}{c}\right) .
$$

We plug this into 4.14 and separate terms based on the powers of $c$. At order $c^{1}$, the problem is to find $Y_{N}$-periodic $u$ and constant $V_{-1}$ that satisfy

$$
\Delta u-\frac{\left\langle\left(e_{N}+\nabla u\right), \nabla^{2} u\left(e_{N}+\nabla u\right)\right\rangle}{\left|e_{N}+\nabla u\right|^{2}}=V_{-1} .
$$

It is easy to verify that the solution is

$$
V_{-1}=0, \quad u(x)=U\left(x_{N}\right),
$$

so that $\nabla u=U^{\prime}\left(x_{N}\right) e_{N}$. We plug this back into 4.15, and then 4.14. At order $c^{0}$, we need to find a $Y_{N}$-periodic $v$ and constant $V_{0}$ that solves

$$
f(x)\left|\left(1+U^{\prime}\right) e_{N}\right|-\left(\Delta v-\left\langle e_{N}, \nabla^{2} v e_{N}\right\rangle\right)=V_{0} .
$$

We recognize the term inside the parenthesis to be the Laplacian in $\mathbb{R}^{N-1}$. Therefore we can eliminate it at each $x_{N}$ by integrating the equation over $Y_{N-1}$ and using the periodicity of $v$ to obtain

$$
\langle f\rangle_{e_{N}}\left(x_{N}\right)\left|\left(1+U^{\prime}\left(x_{N}\right)\right) e_{N}\right|=V_{0},
$$

where we have used the notation introduced earlier. It is clear that $V_{0}$ is determined by this onedimensional problem which is essentially the same as that analyzed in [1, 5].

Let us assume for the moment that either $\langle f\rangle_{e_{N}}>0$ everywhere or $\langle f\rangle_{e_{N}}<0$ everywhere, and seek a solution with $U^{\prime} \geqslant-1$. We can then rewrite (4.16) as

$$
1+U^{\prime}=\frac{V_{0}}{\langle f\rangle_{e_{N}}} .
$$

Integrating with respect to $x_{N}$ and using the periodicity of $U$, we obtain

$$
1=V_{0}\left\langle\langle f\rangle_{e_{N}}^{-1}\right\rangle_{e_{N}^{\perp}} \quad \text { or } \quad V_{0}=\left\langle\langle f\rangle_{e_{N}}^{-1}\right\rangle_{e_{N}^{\perp}}^{-1} .
$$

We thus obtain the desired result that

$$
V=\left\langle\langle f\rangle_{e_{N}}^{-1}\right\rangle_{e_{N}^{\perp}}^{-1}
$$

in the limit as $c \rightarrow \infty$. If $\langle f\rangle_{e_{N}}$ takes both signs, then it is clear from (4.16) that $V_{0}$ has to have both signs and thus must be zero, once again giving us the desired result.

\section{Acknowledgements}

This work was carried out while BC was at the California Institute of Technology, and partially when both $\mathrm{BC}$ and $\mathrm{KB}$ were visiting the Isaac Newton Institute, Cambridge, UK. It is a pleasure to thank Prof. L. C. Evans for useful discussions. We are also grateful for the support of the U.S. National Science Foundation (CMS 9457573) and the Air-Force Office of Scientific Research through a MURI grant (F49602-98-1-0433). 


\section{REFERENCES}

1. Abeyaratne, R., Chu, C., \& James, R. D. Kinetics of materials with wiggly energies: theory and application to the evolution of twinning microstructures in a $\mathrm{Cu}-\mathrm{Al}-\mathrm{Ni}$ shape memory alloy. Philos. Mag. A 73 (1996), 457-497.

2. Alava, M. \& MuÑoz, M. A. Interface depinning versus absorbing-state phase transitions. Phys. Rev. E 65 (2002), Art. 026145.

3. Barles, G., Soner, H. M., \& Souganidis, P. E. Front propagation and phase field theory. SIAM J. Control Optim. 31 (1993), 439-469. Zbl 0785.35049 MR 94c:35005

4. Bensoussan, A., Lions, J. L., \& Papanicolaou, G. Asymptotic Analysis for Periodic Structures. North-Holland (1978). Zbl 0404.35001 MR 82h:35001

5. Bhattacharya, K. Phase boundary propagation in a heterogeneous body. Proc. Roy. Soc. London A Math. 455 (1982), 757-766. Zbl 0990.74047 MR 2000d:74061

6. CAfFARELli, L. A. A Harnack inequality approach to the regularity of free boundaries. I. Lipschitz free boundaries are $C^{1, \alpha}$. Rev. Mat. Iberoamer. 3 (1987), 139-162. Zbl 0676.35085 MR 90d:35306

7. CAffarelli, L. A. A Harnack inequality approach to the regularity of free boundaries. II. Flat boundaries are Lipschitz. Comm. Pure Appl. Math. 42 (1989), 55-78. Zbl 0676.35086 MR 90b:35246

8. CAfFARELli, L. A. A Harnack inequality approach to the regularity of free boundaries. III. Existence theory, compactness and dependence on X. Ann. Scoula Norm. Sup. Pisa Cl. Sci. 15 (1988), 583-602. Zbl 0702.35249 MR 91a:35170

9. Chen, Y.-G., Giga, Y., \& Goto, S. Uniqueness and existence of viscosity solutions of generalized mean curvature flow equations. J. Diff. Geometry 33 (1991), 749-786. Zbl 0696.35087 MR 93a:35093

10. Chen, Y.-G., Giga, Y., \& Goto, S. Remarks on viscosity solutions for evolution equations. Proc. Japan Acad. Ser. A 67 (1991), 323-328. Zbl 0816.35063 MR 93b:35076

11. Craciun, B. \& Bhattacharya, K. Homogenization of a Hamilton-Jacobi equation associated with the geometric motion of an interface. Proc. Roy. Soc. Edinburgh Sect. A 133 (2003), 773-805. MR 2006202

12. Craciun, B. \& Bhattacharya, K. The effect of precipitates on the motion of a twin boundary. In preparation (2002).

13. Crandall, M., Ishit, H., \& Lions, P. L. User's guide to viscosity solutions of second order partial differential equations. Bull. Amer. Math. Soc. 27 (1992), 1-67. Zbl 0755.35015 MR 92j:35050

14. De la Rubia, T. D., Zbib, H. M., Khraishi, T. A., Wirth, B. D., Victoria, M., \& CAtURiA, M. J. Multiscale modelling of plastic flow localization in irradiated materials. Nature 406 (2000), 871-874.

15. E, W. A class of homogenization problems in the calculus of variations. Comm. Pure Appl. Math. 44 (1991), 733-759. Zbl 0773.49007 MR 92h:49010

16. Evans, L. C. Periodic homogenization of certain nonlinear partial differential equations. Proc. Roy. Soc. Edinburgh Sect. A 120 (1992), 245-265. Zbl 0796.35011 MR 93a:35016

17. Ghoniem, N. M., Tong, S. H., \& Sun, L. Z. Parametric dislocation dynamics: A thermodynamicsbased approach to investigations of mesoscopic plastic deformation. Phys. Rev. B 61 (2000), 913-927.

18. GIGA, Y. Surface evolution equations-a level set method. Hokkaido Univ. Technical Report Series in Mathematics 71 (2002).

19. Giga, Y., Goto, S., IshiI, H., \& Sato, M.-H. Comparison principle and convexity preserving properties for singular degenerate parabolic equations on unbounded domains. Indiana Univ. Math. J. 40 (1991), 443-470. Zbl 0836.35009 MR 92h:35010

20. Golestanian, R. \& Raphael, E. Roughening transition in a moving contact line. Phys. Rev. E 67 (2003), Art. 031603. 
21. Gurtin, M. E. Thermomechanics of Evolving Phase Boundaries in the Plane. Oxford Univ. Press (1993). Zbl 0787.73004 MR 97k:73001

22. Halpin-Healy, T. \& Zhang, Y. C. Kinetic roughening phenomena, stochastic growth, directed polymers and all that-Aspects of multidisciplinary statistical mechanics. Phys. Rep. 254 (1995), 215415.

23. Hiratani, M. \& Zвiв, H. M. On dislocation-defect interactions and patterning: stochastic discrete dislocation dynamics (SDD). J. Nucl. Mat. 323 (2003), 290-303.

24. Hirth, J. P. \& Lothe, J. Theory of Dislocations. Krieger (1992).

25. Koslowski, M., Cuitiño, A. M., \& Ortiz, M. A phase-field theory of dislocation dynamics, strain hardening and hysteresis in ductile single crystals. J. Mech. Phys. Solids 50 (2002), 2597-2635. Zbl pre01929473 MR 2003h:74059

26. Lions, P. L., Papanicolaou, G., \& Varadhan, S. R. S. Homogenization of Hamilton Jacobi equation. Preprint (1987).

27. Nicola, L., VAn Der Giessen, E., \& Needlemman, A. 2D dislocation dynamics in thin metal layers. Mat. Sci. Engng. A 309 (2001), 274-277.

28. PARK, K. \& Kim, I. M. Dynamics of an interface driven through random media: The effect of spatially correlated noise. J. Phys. Soc. Japan 72 (2003), 111-116.

29. Petukhov, B. V. Statistical model of the local pinning of dislocations due to cross-slip events. Mat. Sci. Engng. A 309 (2001), 345-347.

30. Sethinan, J. A. Level Set Methods and Fast Marching Methods: Evolving Interfaces in Computational Geometry, Fluid Mechanics, Computer Vision, and Materials Science. Cambridge Univ. Press (1999). Zbl 0973.76003 MR 2000c:65015

31. Sussman, M., Smereka, P., \& Osher, S. A level set approach for computing solutions to incompressible two-phase flow. J. Comput. Phys. 114 (1994), 146-159. Zbl 0808.76077

32. Vannimenus, J. Some recent (and surprising) results on interface and contact line depinning in random media. Phys. A 314 (2002), 264-271. Zbl 0868.35046 